\title{
Disturbed radio and optical morphology in the interacting galaxy pairs
}

\author{
M. Kunert-Bajraszewska ${ }^{1}$, A. Janiuk ${ }^{2}$, M. Hajduk ${ }^{3}$ \\ ${ }^{1}$ Toruń Centre for Astronomy, N. Copernicus University, Gagarina 11, 87-100 Toruń, Poland \\ email: magda@astro.uni.torun.pl \\ ${ }^{2}$ Center for Theoretical Physics, PAS, Al.Lotnikow 32/46, 02-668 Warsaw, Poland \\ ${ }^{3}$ Nicolaus Copernicus Astronomical Center, PAS, Bartycka 18, 00-716 Warsaw, Poland
}

\begin{abstract}
We present high resolution radio observations and their analysis of a radio-loud compact steep spectrum (CSS) sources FIRST J164311.3+315618 and FIRST J150805.6+342323. The radio observations presented here were made with the multi-element radio linked interferometer network (MERLIN) at $1.66 \mathrm{GHz}$ and $5 \mathrm{GHz}$. The radio source FIRST J164311.3+315618 is associated with a small separation, 2.3 arcsec, radio-loud/radio-quiet binary quasar. The radio object FIRST J150805.6+342323 is associated with the north-eastern member of an interacting galaxy pair separated by 24 arcseconds. Our observations revealed disrupted radio morphology of both CSS sources. The distortions of the host galaxy of the two systems are also clearly visible in the optical images and, in the case of binary quasar FIRST J164311.3+315618, are well correlated with the radio ones. We suggest that the ignition of the AGN activity and its complex morphology is caused by the interaction with the companion in the process of merging.
\end{abstract}

Keywords. Physical data and processes: accretion, galaxies: active, galaxies: evolution

\section{Introduction}

There is an ongoing discussion about the mechanism that triggers gas accretion and the nuclear activity in the galaxies. On short timescale, $10^{4}-10^{5}$ years, it can be minor merger or instabilities in the accretion flow (e.g.Czerny et al. (2009)). On long timescales, up to $10^{8}$ years, the major merger activity is broadly considered as the cause of an enhanced accretion flow and the main provider of material for the black hole growth (Barnes \& Hernquist (1996)). If sufficient gas accretes onto both SMBHs in the interacting galaxy pair, each may be visible as an active galactic nucleus (AGN). Though, only about $1 \%$ of interacting galaxies has an AGN present. This can be explained in different ways: 1) the active nucleus can be buried beneath thick layers of gas and dust, 2) activity can be triggered on only one of the two black holes, and 3) there is a time delay between the onset of activity in the two merging systems (Foreman et al. (2009)). To be identified as an AGN, the source needs to possess unique spectral signatures, emitted by broad/narrow emission regions, the accretion disk and the jet. The X-ray, infrared and radio detection provide further confirmation of an AGN activity in a galaxy. Here we report the AGN detection in the two interacting systems.

\section{Discussion and Results}

Both discussed sources, FIRST J164311.3+315618 and FIRST J150805.6+342323, belong to the sample of Compact Steep Spectrum (CSS) sources we observed and analysed in Kunert-Bajraszewska et al. (2010). CSS sources form a well defined class of compact radio objects $(\leqslant 20 \mathrm{kpc})$ and are considered to be younger progenitors of large radio-loud 

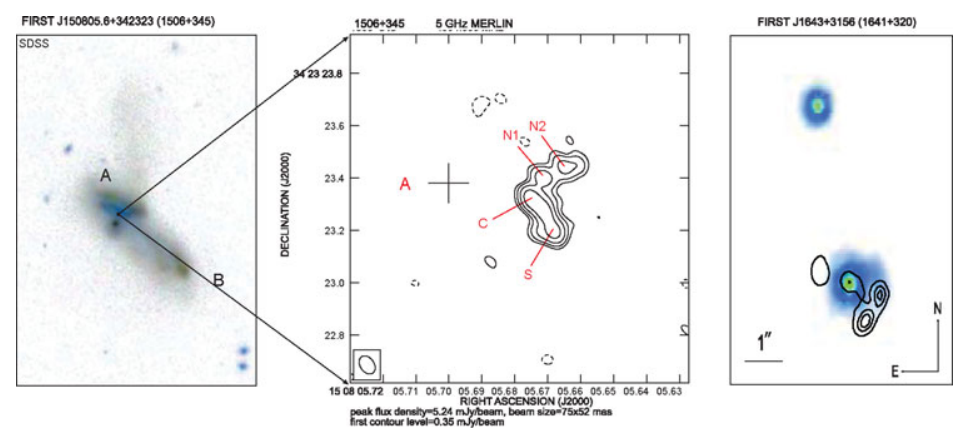

Figure 1. FIRST J150805.6+342323: SDSS image (left), $5 \mathrm{GHz}$ MERLIN image (middle). The HST/ACS image of FIRST J1643+3156 in F814W filter with an overlay of contours of the radio emission at $1.66 \mathrm{GHz}$ (right). Contours increase by a factor 2, and the first radio contour level corresponds to $3 \sigma$. The cross indicates the position of an optical counterpart.

AGNs. We have made observations of the sample with MERLIN at $1.66 \mathrm{GHz}$ and $5 \mathrm{GHz}$ and found that the morphologies of the binary systems are highly disrupted and do not resemble typical CSS sources with symmetric double lobes.

FIRST J164311.3+315618 $(1641+320, z=0.586)$ is a binary quasar and its optical and radio distortions are well correlated (Fig.1, Kunert-Bajraszewska \& Janiuk (2011)).

FIRST J150805.6+342323 $(1506+345, z=0.045)$ is an interacting galaxy pair. The CO line observations made by Mazzarella et al. (1993) revealed that each component of this pair contain $\sim 10^{10} M_{\odot}$ of molecular gas and the authors suggest that this pair will evolve into gas-rich galaxy. The spatially resolved NTT spectrum of the galaxy pair show narrow emission lines imposed on the continuum. The [NII] $\lambda 6584$ to $\mathrm{H} \alpha$ line ratio is typical for a star-forming galaxy and increases toward center. This may reflect the abundance gradient or result from contamination by the nuclear emission close to the radio source. The optical disrupted structure of FIRST J150805.6+342323 is evident in SDSS image. The radio emission is associated with the north-eastern member $A$ of this galaxy pair (Fig. 1). Our $5 \mathrm{GHz}$ radio observations performed by MERLIN interferometer show complicated structure resolved into four components. The compact component indicated as $C$ is probably a radio core, components $S, N 1$ and $N 2$ are parts of the jet/lobes. The probable initial direction of the radio jet (weak components $N 1$ and N2) could be changed by the accretion disk precession due to tidal torques induced by the companion in this galaxy pair. The components $C$ and $S$ probably shows the current jet direction.

Based on the analysis performed on the observed by us galaxy pairs we suggest that the distortions of the radio structure visible in the binary system are short-lived phenomena. The disturbed radio components that are no longer powered by the jet can quickly fade away. This makes the potential distortions detectable only during a short period of time and implies a low detection rate.

\section{References}

Barnes, J. E. \& Hernquist, L., 1996, ApJ, 471, 115

Czerny, B., Siemiginowska, A., Janiuk, A., et al., 2009, ApJ, 698, 840

Foreman, G., Volonteri, M., \& Dotti, M., 2009, ApJ, 693, 1554

Kunert-Bajraszewska, M., Janiuk, A., Gawroński, M. P., \& Siemiginowska, A., 2010, ApJ, 718, 1345

Kunert-Bajraszewska M. \& Janiuk A., 2011, ApJ, 736, 125

Mazzarella, J. M., Graham, J. R., Sanders, D. B., \& Djorgovski, S., 1993, ApJ, 409, 170 